\title{
PRETEXT Stage 1 Hepatoblastoma
}

National Cancer Institute

\section{Source}

National Cancer Institute. PRETEXT Stage 1 Hepatoblastoma. NCI Thesaurus. Code

C7139.

An internationally developed presurgical anatomic staging system using imaging techniques: Tumor involves only 1 quadrant; 3 adjoining liver quadrants are free of tumor. (from PDQ 2004) 